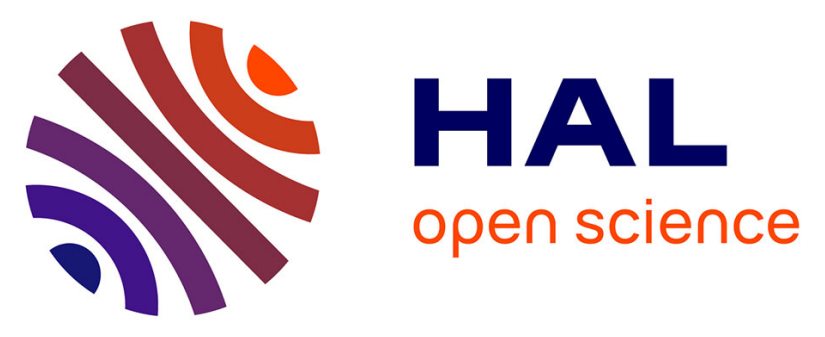

\title{
3D aortic morphology and stiffness in MRI using semi-automated cylindrical active surface provides optimized description of the vascular effects of aging and hypertension
}

Thomas Dietenbeck, Damian Craiem, David Rosenbaum, Alain Giron, Alain de Cesare, Kévin Bouaou, Xavier Girerd, Philippe Cluzel, Alban Redheuil, Nadjia Kachenoura

\section{- To cite this version:}

Thomas Dietenbeck, Damian Craiem, David Rosenbaum, Alain Giron, Alain de Cesare, et al.. 3D aortic morphology and stiffness in MRI using semi-automated cylindrical active surface provides optimized description of the vascular effects of aging and hypertension. Computers in Biology and Medicine, 2018, 103, pp.101-108. 10.1016/j.compbiomed.2018.10.006 hal-01976985

\section{HAL Id: hal-01976985 \\ https://hal.sorbonne-universite.fr/hal-01976985}

Submitted on 10 Jan 2019

HAL is a multi-disciplinary open access archive for the deposit and dissemination of scientific research documents, whether they are published or not. The documents may come from teaching and research institutions in France or abroad, or from public or private research centers.
L'archive ouverte pluridisciplinaire HAL, est destinée au dépôt et à la diffusion de documents scientifiques de niveau recherche, publiés ou non, émanant des établissements d'enseignement et de recherche français ou étrangers, des laboratoires publics ou privés. 


\section{D aortic morphology and stiffness in MRI using semi-automated}

\section{cylindrical active surface provides optimized description of the}

\section{vascular effects of aging and hypertension}

Thomas Dietenbeck, $\mathrm{PhD}^{1,}{ }^{*}$, Damian Craiem, $\mathrm{PhD}^{2}$, David Rosenbaum, MD, PhD ${ }^{1,3}$, Alain Giron, $\mathrm{PhD}^{1}$, Alain De Cesare, $\mathrm{PhD}^{1}$, Kévin Bouaou ${ }^{1}$, Xavier Girerd, $\mathrm{MD}^{3}$, Philippe Cluzel, MD, PhD ${ }^{1,3,4}$, Alban Redheuil, MD, PhD ${ }^{1,3,4}$, Nadjia Kachenoura, $\mathrm{PhD}^{1}$

${ }^{1}$ Sorbonne Université, INSERM, CNRS, Laboratoire d'Imagerie Biomédicale (LIB), F-75006, Paris, France

${ }^{2}$ Instituto de Medicina Traslacional, Trasplante y Bioingeniería (IMeTTyB), Universidad FavaloroCONICET, Buenos Aires, Argentina.

${ }^{3}$ ICAN Institute of Cardiometabolism and Nutrition, Imaging Core Lab, Paris, France

${ }^{4}$ Département d'imagerie cardiovasculaire DICVRIT, Institut de Cardiologie, Groupe Hospitalier Pitié Salpêtrière APHP, Paris, France

* Corresponding author

Address : UPMC - Campus des Cordeliers

15 rue de l'Ecole de Médecine, 75006 PARIS (FRANCE)

Phone number: +33144279119

Email address: thomas.dietenbeck@upmc.fr 


\section{Abstract}

\section{Background}

Clinically, aortic geometry assessment is mainly based on the measurement of maximal diameters at different anatomic locations, which are subsequently used to indicate prophylactic aortic surgery. However, 3D evaluation of aortic morphology could provide volumetric quantification, which integrates both aortic dilatation and elongation and might thus be more sensitive to early geometric changes than diameters. Precise aortic morphology is also required for the calculation of pulse wave velocity $\left(P W V_{M R I}\right)$, an established marker of aortic stiffness. Accordingly, we proposed a 3D semiautomated analysis of thoracic aorta MRI data optimizing morphological and subsequent stiffness assessment.

\section{Methods}

We studied 74 individuals (40 males, 50 \pm 12 years): 21 healthy volunteers and 53 patients with hypertension in whom aortic 3D MRI angiography and 2D+t phase-contrast and cine imaging were performed. A semi-automated method was proposed for volumetric aortic segmentation and was evaluated by studying resulting measurements (length, diameters, volumes and $P W V_{M R I}$ ) in terms of: 1) reproducibility, 2) correlations with well-established 2D aortic length and diameters, 3) associations with age, carotid-femoral PWV (cf-PWV) and presence of hypertension.

\section{Results}

The measurements obtained with the proposed method were reproducible (coefficients of variation $\leq 5.1 \%$ ) and were highly correlated with 2D measurements (arch length: $r=0.80$, Bland-Altman mean bias [limits]: $\left.2.7 \mathrm{~mm}[-25 ; 30] ; \mathrm{PWV}_{\mathrm{MRI}} \mathrm{r}=0.95,0.22 \mathrm{~m} / \mathrm{s}[-1.9 ; 2.4]\right)$. Higher or similar correlations with age were found for the proposed 3D method compared to the 2D approach (arch length: $r=$ $\left.0.47(2 \mathrm{D}), r=0.60(3 \mathrm{D}) ; \mathrm{PWV}_{\mathrm{MRI}}: r=0.63(2 \mathrm{D}), r=0.64(3 \mathrm{D})\right)$. Moreover, a significant association was found between $P W V_{M R I}$ and cf-PWV $(r=0.49, p<0.001)$. All aortic measurements increased with hypertension $(p<0.05)$ and with age: arch length $(+9 \mathrm{~mm} /$ decade); diameters: ascending 
$(+1.2 \mathrm{~mm} /$ decade) and descending aorta ( $+1.0 \mathrm{~mm} /$ decade); volumes: ascending $(+2.6 \mathrm{~mL} /$ decade) and descending aorta $\left(+4.0 \mathrm{~mL} /\right.$ decade); $\operatorname{PWV}_{\text {MRI }}\left(+1.7 \mathrm{~m} \cdot \mathrm{s}^{-1} /\right.$ decade).

\section{Conclusions}

A semi-automated method based on cylindrical active surfaces was proposed for the 3D segmentation of the aorta using a single MRI dataset, providing aortic diameters at anatomical landmarks, aortic volumes and the aortic centerline length used for PWV estimation. Such measurements were reproducible and comparable to expert measurements, which required timeconsuming centerline delineation. Furthermore, expected relationships with age and hypertension were found indicating the consistency of our measurements.

\section{Keywords}

Aorta, 3D cardiovascular magnetic resonance imaging, segmentation, active contour, aortic geometry, aortic stiffness 


\section{Background}

Age and aggravating conditions, such as hypertension and cardiometabolic disease, have a deleterious effect on aortic structure, geometry, function and hemodynamics that can be measured through changes in aortic arch length, diameters, wall stiffness, distension and pressures, as well as circulating blood flow. Such arterial alterations are associated with left ventricular hypertrophic remodeling $[1,2]$ and increased mortality $[3,4]$. Identifying early stages of aortic remodeling could help in preventing adverse cardiovascular events.

Over the last decades, cardiovascular magnetic resonance imaging (MRI) has been proposed to study the central aorta due to its ability to non-invasively assess aortic anatomy [5], geometry $[1,6,7]$, function [8-13] and flow [14-17]. For aortic morphological evaluation, 3D steady-state free precession (SSFP) magnetic resonance with ECG and respiratory navigator gating provides high resolution isotropic angiography of the thoracic aorta with or without contrast agents. 3D aortic segmentation allows the computation of $3 \mathrm{D}$ volumetric indices which, by integrating both aortic dilatation and elongation, may be more sensitive to assess geometric changes than diameters. In addition, a 3D segmentation can provide the aortic arch length needed for surgical or interventional planning or the computation of aortic Pulse Wave Velocity (PWV). PWV is an established arterial stiffness biomarker and its assessment requires an accurate estimation of the distance between two arterial locations and the transit time of the flow wave travelling between these two locations. Although several automated techniques have been proposed to estimate the transit time from phase-contrast (PC) MRI $[11,12]$ using the ascending (AAo) and descending (DAo) aortic flow curves, the aortic arch length is usually estimated manually.

In this work, we described a new semi-automated segmentation technique based on explicit active contours previously used for the 3D segmentation of the heart [18]. We proposed an adaptation of the evolving surface formulation to the tubular structure of the aorta and to the surrounding intensity inhomogeneity. Such 3D segmentation of aortic MRI then allowed the automatic 
measurement of aortic arch length, diameters at predefined landmarks and segmental volumes as well as the calculation of $\mathrm{PWV}_{\mathrm{MRI}}$. The reproducibility of our measurements, their dependence on spatial resolution, relationship with relevant and established determinants such as age and hypertension and the correlation with tonometric cf-PWV were studied to compare the respective ability of diameters and volumes to detect early aortic alterations.

\section{Materials and methods}

\subsection{Data acquisition}

We studied 21 normotensive healthy volunteers (43 \pm 11 years, 8 females), 26 controlled hypertensive patients (age $52 \pm 12$ years, 14 females), and 27 uncontrolled hypertensive patients (53 \pm 12 years, 12 females) prospectively recruited in a dedicated outpatient clinic (Cardiovascular Prevention Unit). Clinical evaluations were carried out according to the principles outlined in the Declaration of Helsinki. Approval of the local Ethics Committee was obtained and informed consent was signed by all participants.

All patients underwent MRI on a 1.5 Tesla (Aera, Siemens HealthCare, Erlangen, Germany) magnet with cardiac phased-array coil and ECG-gated sequences. 3D steady-state free precession (SSFP) images were acquired in a sagittal-oblique view to cover the whole thoracic aorta. Acquisition was performed during the diastolic phase with ECG gating and respiratory navigator gating after the injection of $0.1 \mathrm{mM}^{-\mathrm{kg}^{-1}}$ of contrast agent (Gadobenate dimeglumine, MultiHance, Bracco). Average scan parameters were: repetition time $=285.2 \mathrm{~ms}$, echo time $=1.5 \mathrm{~ms}$, flip angle $=90^{\circ}$, pixel size $=$ $0.64 \mathrm{~mm} \times 0.64 \mathrm{~mm}$, slice thickness $=1 \mathrm{~mm}$.

Additionally, PC images of the proximal aorta in a single axial view at the level of the bifurcation of the pulmonary trunk, perpendicular to the ascending and descending aorta, were acquired during breath holds using a retrospectively ECG-gated sequence with a velocity encoding gradient in the through-plane direction. This acquisition provided phase-related pairs of modulus and velocityencoded images. Average scan parameters were: repetition time $=53.5 \mathrm{~ms}$, echo time $=3.23 \mathrm{~ms}$, flip 
angle $=30^{\circ}$, pixel size $=1.72 \mathrm{~mm} \times 1.72 \mathrm{~mm}$, slice thickness $=6 \mathrm{~mm}$, and encoding velocity $=200$ $\mathrm{cm} / \mathrm{s}$. At this same anatomical location, a cine SSFP dataset was also acquired using the following average scan parameters: repetition time $=32.9 \mathrm{~ms}$, echo time $=1.82 \mathrm{~ms}$, flip angle $=74^{\circ}$, pixel size $=$ $1 \mathrm{~mm} \times 1 \mathrm{~mm}$, slice thickness $=6 \mathrm{~mm}$.

Central blood pressures were recorded using the SphygmoCor Xcel device (AtCor) during the MRI aortic acquisitions (average of 3 measurements).

\subsection{D segmentation process}

The segmentation process comprised 3 main steps: user initialization, centerline extraction and 3D aortic segmentation. First, the user was asked to manually define 7 anatomical landmarks along the center of the aorta (sino-tubular junction, AAo and DAo at the location used for phase contrast and cine acquisitions, brachiocephalic trunk, left subclavian artery, diaphragm and celiac trunk), as shown in Fig.1(a). Second, the aortic centerline was extracted from the rough skeleton of the aorta from a thresholded 3D volume [19]. The centerline corresponded to the shortest path joining two consecutive landmarks and going through the skeleton [20] (Fig.1(b)).

Third, we adapted an active contour method recently proposed for the 3D segmentation of the heart [18] to the tubular morphology of the aorta. The idea behind this technique was to represent the evolving boundary by an explicit function, where one coordinate of a point was expressed as a function of the remaining coordinates. Formally, given a point $\mathbf{x}=\left(x_{1}, x_{2}, \ldots, x_{n}\right) \in \mathrm{i}^{n}$ in a $\mathrm{n}$ dimensional space, the explicit active contour was defined as

$$
\psi: i^{n-1} \mathrm{a} i,\left(x_{2}, x_{3}, \ldots, x_{n}\right) \text { a } x_{1}=\psi\left(x_{2}, x_{3}, \ldots, x_{n}\right),
$$

where $\psi$ is the explicit function represented as a linear combination of B-Splines functions

$$
\psi\left(\mathbf{X}^{*}\right)=\sum_{\mathbf{k} \in \dot{\phi}^{n-1}} c[\mathbf{k}] \beta^{3}\left(\frac{\mathbf{X}^{*}}{h}-\mathbf{k}\right),
$$


$\beta^{3}$ is a uniform symmetric $(\mathrm{n}-1)$ dimensional cubic $\mathrm{B}$-spline, $\mathrm{c}$ gathers the interpolation coefficients of the B-spline representation of $\psi$ and $h$ is a scaling factor defining the spacing on a rectangular grid between the B-spline knots $\mathbf{k}$. Thus, $h$ acts as a smoothing parameter of the segmentation results.

Given the tubular nature of the aorta, we proposed to represent the aortic surface using cylindrical coordinates $\mathbf{x}=(\mathbf{s}, \theta, \rho)$ and expressed the aortic radius as $\rho=\psi(\mathbf{s}, \theta)$ where $\mathbf{s}$ is the 3D coordinates of a centerline point and $\theta$ the angular position in a plane perpendicular to the centerline axis.

The surface then evolved towards the aorta's boundaries through gradient descent by minimizing

$$
E(\psi)=E_{d}(\psi)+\lambda_{r} E_{r}(\psi)
$$

where $E_{d}$ is a data attachment term, $E_{r}$ is a regularization term and $\lambda_{r}$ is a weight balance between the two energies.

To handle heterogeneities inside the aorta, a localized energy $E_{d}[21]$ guided the surface to areas where the difference between the inside and outside mean intensity was maximal. These means were computed in small neighborhoods around each point of the surface. In regions with a high centerline curvature (such as the aortic arch), 2 radii might cross, leading to surface selfintersections. To avoid this behavior, a regularization term $E_{r}$ was added, minimizing the surface mean curvature [22] to the data attachment term.

After the segmentation, the centerline was automatically repositioned at the center of the segmented volume; the aortic arch length was then computed as the centerline length between the AAo and DAo landmarks and the AAo and DAo diameters as twice the mean of $\psi$ at the corresponding landmarks. AAo volume was defined as the volume between the STJ and the BCA and DAo volume as the volume between the LCA and the diaphragm.

The parameters of the method were set as follows: the surface was discretized by 64 points along the aortic centerline and 32 angular positions; the scaling factor $h$ was set to 1 and the weight $\lambda_{r}$ to 1 . A $3 \mathrm{D}$ rendering of the segmentation result along with the aorta centerline are shown in Fig.1(c). The 


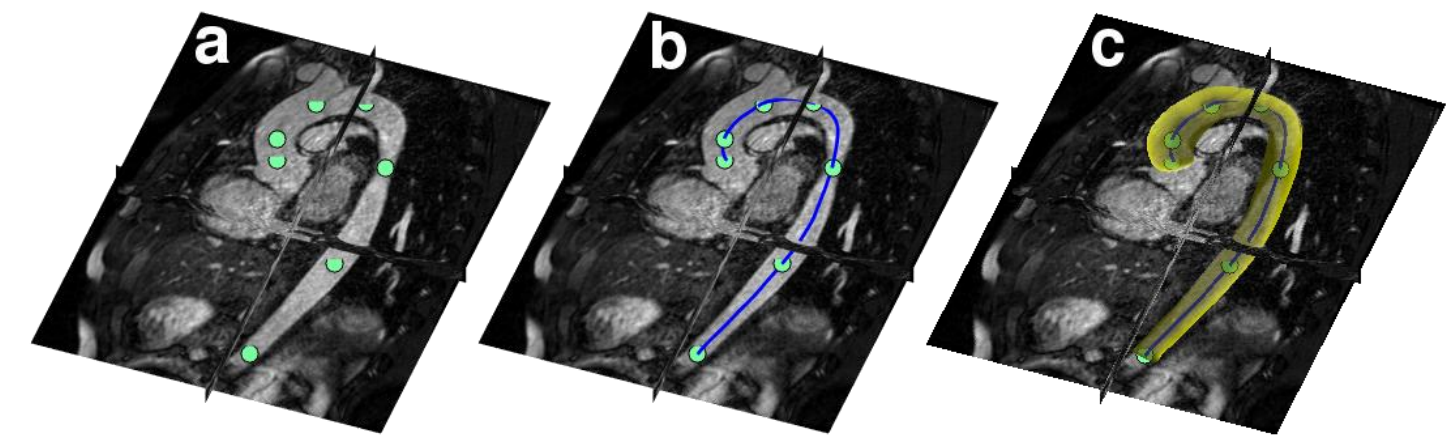

Figure 1: Manual positioning of 7 anatomical landmarks (a) which are used to extract the aorta centerline (b). (c) 3D visualization of the segmented aorta.

total average analysis time was around $5 \mathrm{~min}(2-3 \mathrm{~min}$ to load the data and to set the landmarks and 2 min for the centerline extraction and the 3D aortic segmentation) for a Matlab (The Mathworks Inc., Natick, MA) implementation of the software.

\subsection{Measures used for comparisons}

A manual estimation of the aortic arch length, commonly used for $\mathrm{PWV}_{\mathrm{MRI}}$ estimation, was performed using a previously established procedure $[2,9,23]$ implemented in the ARTFUN software (Laboratoire d'Imagerie Biomédicale, Sorbonne Université, INSERM, CNRS). Briefly, an operator was asked to manually place 6 to 8 landmarks between the AAo and DAo in the center of the aortic lumen, while using the same temporal phase on both axial and sagittal oblique $2 \mathrm{D}$ cine slices. The first and last points were placed at the center of the AAo and DAo in the plane used for PC-MRI and cine axial acquisitions. The 3D coordinates of the positioned markers were recorded and a spline interpolation was performed between them to construct the 3D aortic centerline curve providing the arch length. As multiple 2D axial and sagittal oblique views were considered, this procedure allowed measuring the aortic arch length similar to 3D, taking into account the aortic tortuosity. The ARTFUN software $[24,25]$ was also used to automatically measure: 1 ) the AAo and DAo diameters from 2D cine SSFP images at the same cardiac phase as the 3D acquisition as well as 2) the flow wave transit time (TT) between $A A o$ and DAo used for $P W V_{M R I}$ calculation ( $P W V_{M R I}=$ aortic arch length/TT). An 
illustration of the $2 \mathrm{D}$ automated segmentation of the aortic lumen from $2 \mathrm{D}$ cine $\mathrm{MRI}$ is provided in the supplementary material.

Finally, to mimic lower spatial resolutions such as those of data acquired using 4D-flow MRI, we subsampled our data by a factor of 2 or 3 and studied the segmentation accuracy using the Dice coefficient and the measurements variations between subsampled and full resolution analyses. Such evaluation might be of interest, since one of the important current application is to progress towards $3 D+t$ segmentation of $4 \mathrm{D}$ flow MRI to be able to accurately extract relevant hemodynamic indices in addition to geometrical indices.

\subsection{Statistical analysis}

For both methods, aortic arch length, $\mathrm{PWV}_{\mathrm{MR}}, \mathrm{AAO}$ and $\mathrm{DAo}$ diameters were computed and provided as mean \pm standard deviation (SD). For the proposed method, AAo and DAo volumes were also measured. Linear regression analysis and Bland Altman (BA) plots were performed to compare the proposed method with ARTFUN. Pearson correlation coefficients ( $r$ ) as well as BA mean bias and limits of agreement were provided. A non-parametric Kruskal-Wallis test was conducted to evaluate the differences across methods.

To study the reproducibility of the proposed method, the same user analyzed the whole datasets twice and 4 months apart and the intra-observer variability was assessed using the mean relative error and the coefficient of variation, calculated as the standard deviation of the differences between two measurements divided by their mean.

Correlation coefficients for linear regressions between aortic measures and age or between $\mathrm{PWV} \mathrm{VRI}_{\mathrm{MI}}$ and the reference carotid-femoral PWV (cf-PWV) were also provided. For comparisons between normotensive individuals and controlled or uncontrolled hypertensive patients, a non-parametric Wilcoxon test was used. All reported p-values were two-sided and statistical significance was indicated by $p$-value $<0.05$. 

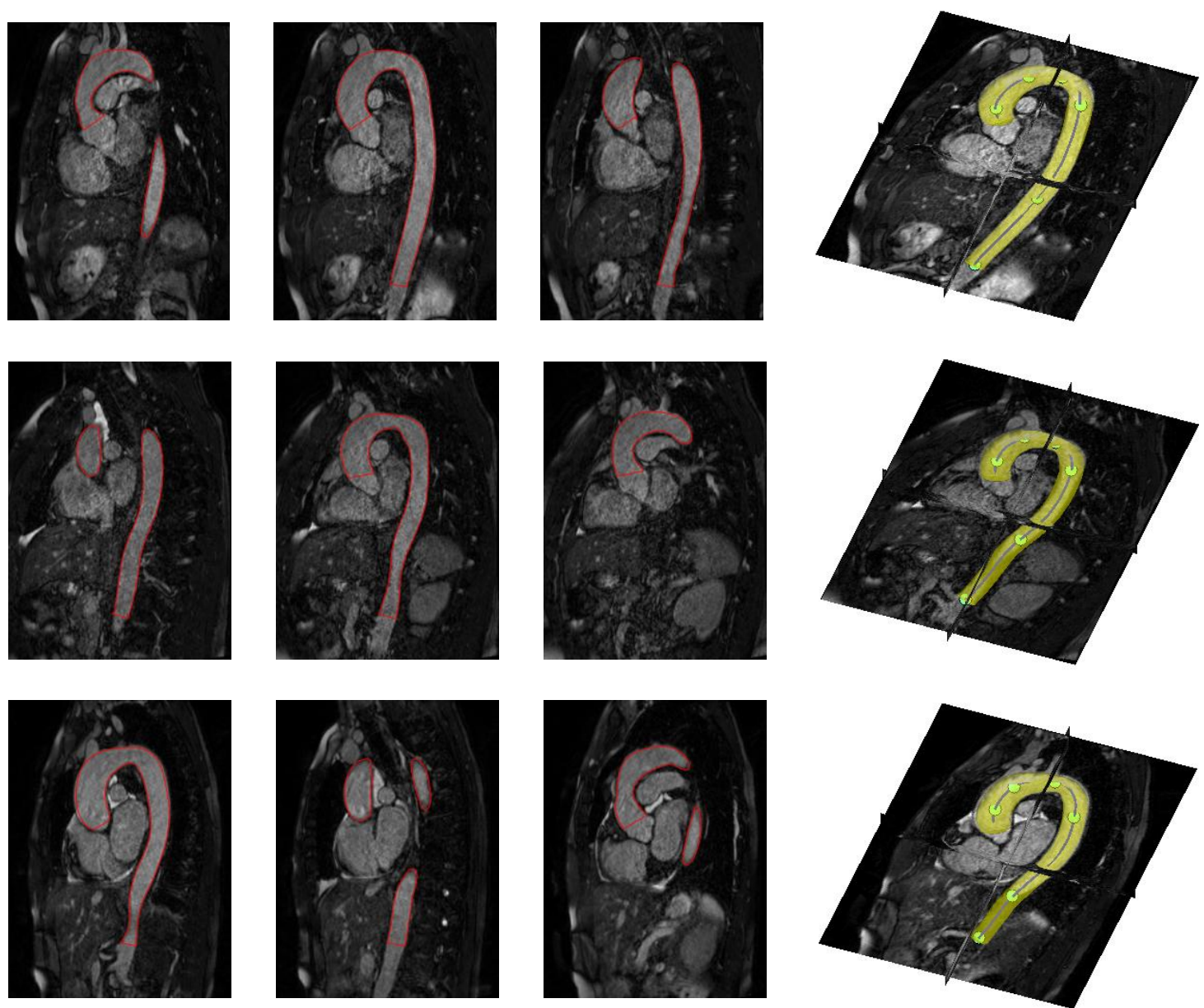

Figure 2: Segmentation results on a healthy volunteer (1rst row), a controlled ( 2 nd row) and an uncontrolled hypertensive (3rd row). For each row, 3 slices of the volume are shown along with the 3D rendering of the aortic surface.

\section{Results}

\subsection{Study population}

Patient characteristics, blood pressures and cf-PWV along with 2D and 3D aortic measurements are summarized in Table 1 for the whole population and separately for the 3 groups: normotensives, controlled hypertensives and uncontrolled hypertensives. Age was slightly higher in the hypertensive patients compared to controls $(\mathrm{p}<0.05)$. As expected, central blood pressures as well as cf-PWV were significantly higher in hypertensives as compared to normotensive subjects $(p<0.001)$. MRI aortic measurements revealed a significant elongation and dilation of the aorta with hypertension $(p<0.05)$. 


\begin{tabular}{ccccc}
\hline All & Normotensives & Controlled & Uncontrolled & p-value \\
& & & & \\
& & & & \\
74 & 21 & 26 & 27 & \\
& & & & \\
$40 / 35$ & $13 / 8$ & $12 / 14$ & $15 / 13$ & \\
$50.2 \pm 12.7$ & $43.5 \pm 11.8$ & $52.0 \pm 12.6^{*}$ & $53.4 \pm 12.6^{*}$ & 0.02 \\
$25.5 \pm 4.2$ & $23.8 \pm 3.0$ & $25.6 \pm 3.7$ & $26.8 \pm 5.0^{*}$ & 0.034 \\
$1.83 \pm 0.22$ & $1.80 \pm 0.21$ & $1.85 \pm 0.22$ & $1.84 \pm 0.24$ & 0.713 \\
$124.3 \pm 14.5$ & $110.7 \pm 13.0$ & $122.4 \pm 9.8^{*}$ & $134.2 \pm 12.4^{*}$ & $<0.001$ \\
$85.5 \pm 10.7$ & $76.8 \pm 9.5$ & $83.8 \pm 8.0^{*}$ & $92.1 \pm 9.8^{*}$ & $<0.001$ \\
$39.6 \pm 9.4$ & $36.1 \pm 8$. & $39.3 \pm 10.9$ & $42.8 \pm 8.3^{*}$ & 0.012 \\
$11.1 \pm 1.8$ & $9.9 \pm 1.4$ & $11.2 \pm 1.7^{*}$ & $11.8 \pm 1.7^{*}$ & $<0.001$
\end{tabular}

\section{Proposed 3D method}

$\begin{array}{cccccc}\text { Arch length }(\mathrm{mm}) & 130.1 \pm 19.8 & 121.5 \pm 17.6 & 128.6 \pm 18.0 & 138.3 \pm 20.6^{*} & <0.01 \\ \text { AAo diameter }(\mathrm{mm}) & 27.8 \pm 3.2 & 25.7 \pm 2.7 & 27.8 \pm 3.1^{*} & 29.3 \pm 2.8^{*} & <0.001 \\ \text { DAo diameter }(\mathrm{mm}) & 20.4 \pm 2.8 & 18.8 \pm 2.3 & 20.8 \pm 2.9^{*} & 21.3 \pm 2.5^{*} & <0.005 \\ \text { AAo volume }(\mathrm{mL}) & 28.9 \pm 10.6 & 22.3 \pm 6.7 & 29.6 \pm 12.0^{*} & 33.4 \pm 9.1^{*} & <0.001 \\ \text { DAo volume }(\mathrm{mL}) & 49.7 \pm 18.2 & 41.3 \pm 11.9 & 51.3 \pm 22.0 & 54.8 \pm 16.4^{*} & <0.05 \\ \quad \text { PWV }_{\text {MRI }}(\mathrm{m} / \mathrm{s}) & 7.29 \pm 3.41 & 5.13 \pm 2.10 & 7.60 \pm 3.31^{*} & 8.84 \pm 3.59^{*} & <0.001 \\ \quad \text { ARTFUN } & & & & \\ \text { Arch length }(\mathrm{mm}) & 127.4 \pm 22.8 & 118.1 \pm 17.7 & 125.7 \pm 23.9 & 136.2 \pm 22.8^{*} & <0.05 \\ \text { AAo diameter }(\mathrm{mm}) & 29.7 \pm 3.9 & 26.8 \pm 3.3 & 30.0 \pm 3.3^{*} & 31.5 \pm 3.5^{*} & <0.001 \\ & & & & \\ \text { DAo diameter }(\mathrm{mm}) & 21.7 \pm 3.3 & 19.7 \pm 3.0 & 21.9 \pm 3.3^{*} & 23.1 \pm 2.9^{*} & <0.005 \\ \text { PWV }_{\text {MRI }}(\mathrm{m} / \mathrm{s}) & 7.16 \pm 3.35 & 5.0 \pm 2.09 & 7.19 \pm 2.83^{*} & 8.95 \pm 3.71^{*} & <0.001\end{array}$

Table 1: Subject characteristics for the whole population and for each group. $p$ values for ANOVA across groups. ${ }^{*}$ means $p<$ 0.05 for comparisons against normotensives 


\subsection{Reproducibility of the 3D approach and its correlation with 2D measures}

Table 2 summarizes the Pearson correlation coefficients ( $r$ ) and the $p$-values as well as the mean bias and limits of agreement from the BA plots for the comparison of the 3D and the 2D measures when available. The corresponding linear regression and BA plots are shown in Fig.3 and Fig.4, respectively. Good correlations were found between the 2 methods for all measurements $(r>0.76, p<0.001)$. In addition, low differences in mean values were found for the aortic arch length (2.1\%), the AAo (6.4\%) and the DAo (6\%) diameters and the $\mathrm{PWV}_{\mathrm{MRI}}(1.8 \%)$, with a statistical significance reached for differences in aortic diameters $(p<0.01)$.

a)

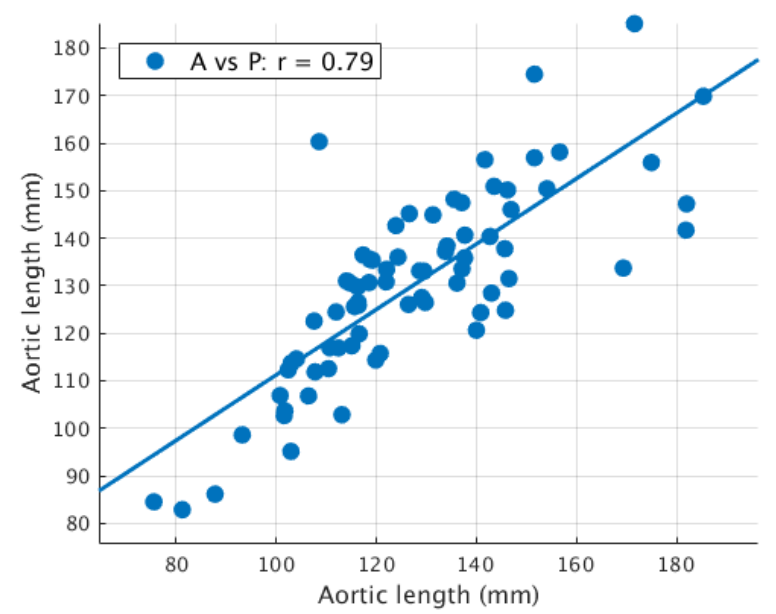

c)

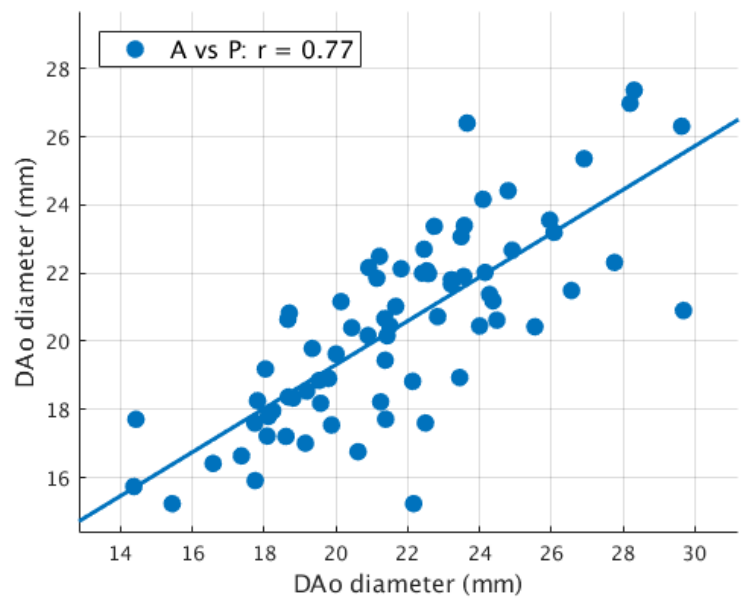

b)

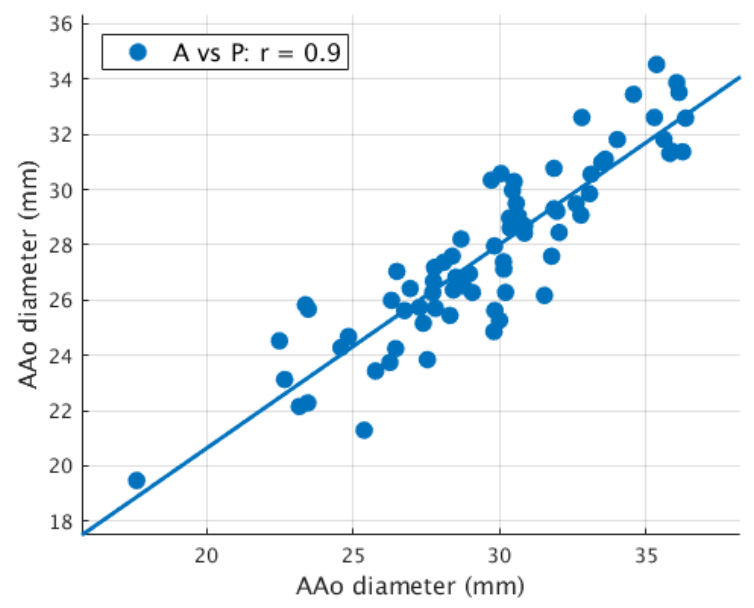

d)

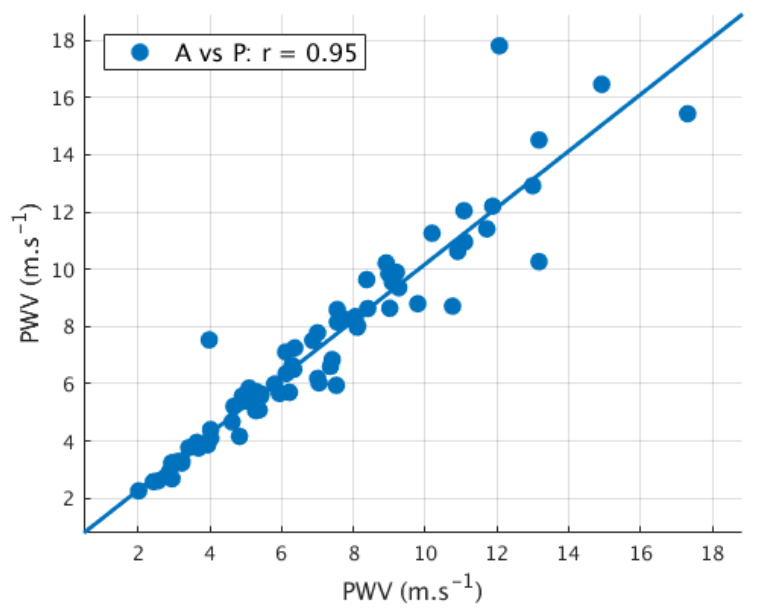

Figure 3: Correlation between the ARTFUN measurements $(A)$ and the proposed method $(P)$ in terms of (a) aortic arch length, aortic diameters in the (b) ascending (AAo) and (c) in the descending aorta (DAo) and (d) PWV $\mathrm{MRI}_{\text {. }}$ 
a)

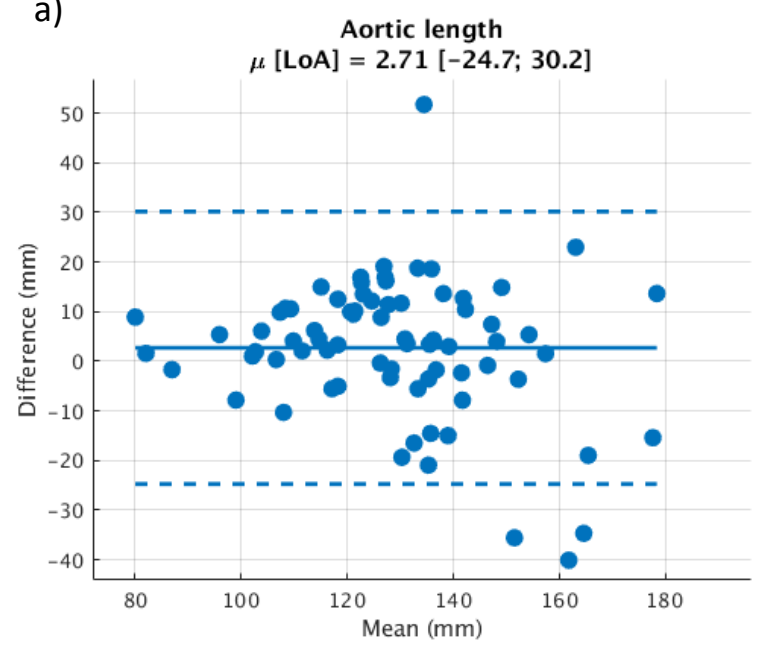

c)

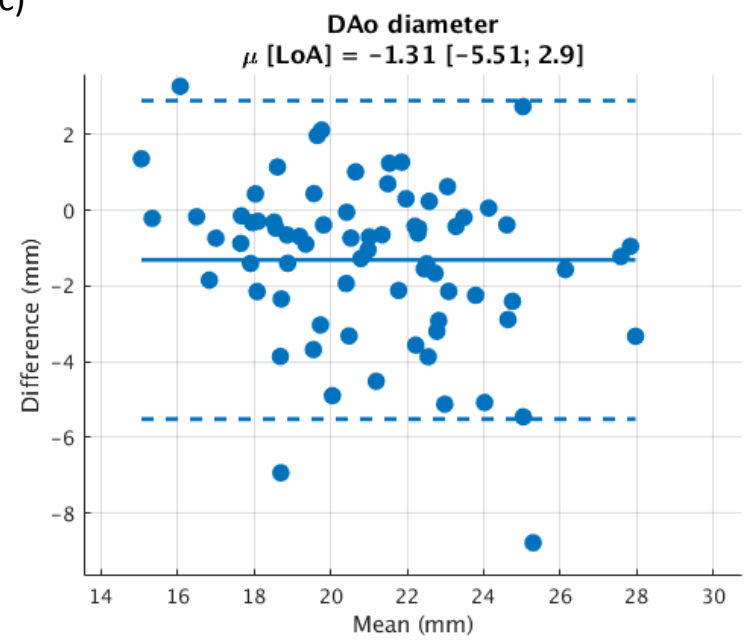

b) AAo diameter

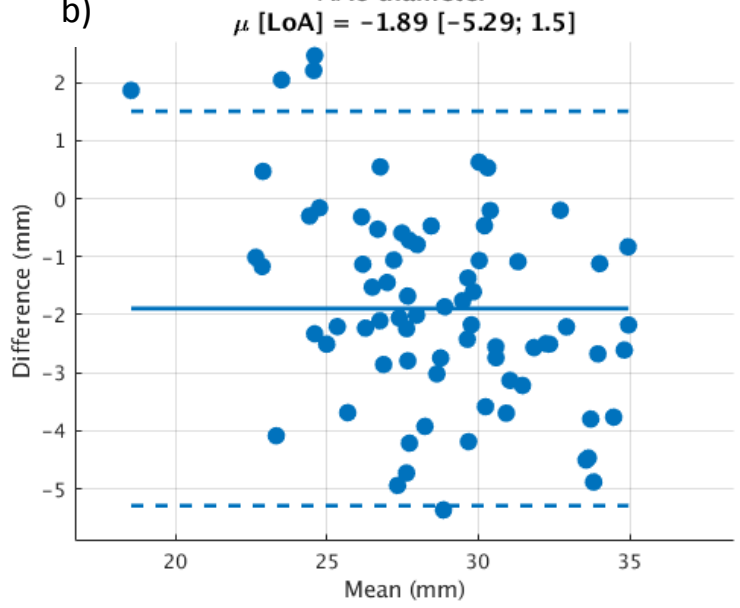

d)

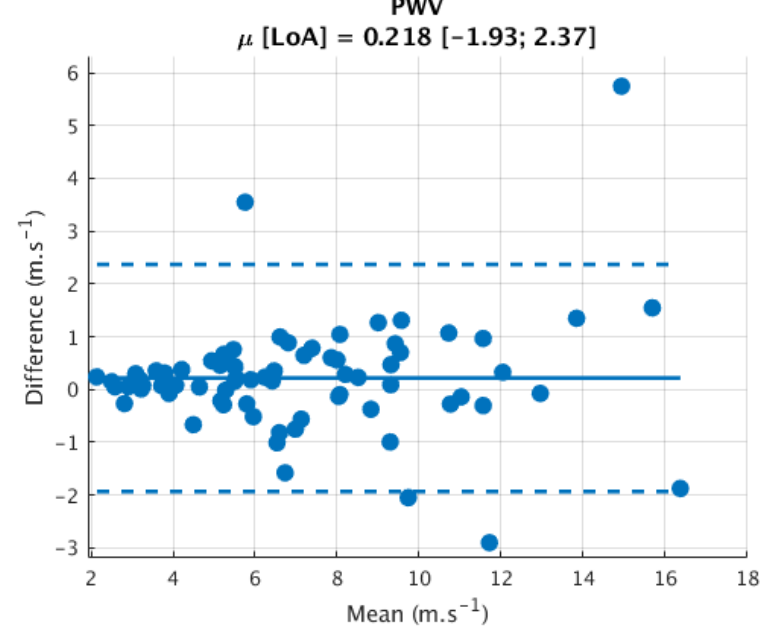

Figure 4: Bland-Altman plots between the ARTFUN measurements $(A)$ and the proposed method $(P)$ in terms of (a) aortic arch length, aortic diameters in the (b) ascending (AAO) and (c) in the descending aorta (DAo) and (d) PWV $\mathrm{MRI}_{\text {- }}$

Correlation coefficient $\quad$ p-value $\quad$ Bland-Altman analysis

\begin{tabular}{cccc}
\hline Arch length $(\mathrm{mm})$ & $r=0.79$ & 0.25 & $2.7[-24.7 ; 30.2]$ \\
AAo diameter $(\mathrm{mm})$ & $r=0.90$ & $<0.001$ & $-1.89[-5.29 ; 1.50]$ \\
DAo diameter $(\mathrm{mm})$ & $r=0.77$ & $<0.01$ & $-1.31[-5.51 ; 2.90]$ \\
$\operatorname{PWV}_{\text {MRI }}(\mathrm{m} / \mathrm{s})$ & $r=0.95$ & 0.73 & $0.22[-1.93 ; 2.37]$
\end{tabular}

Table 2: Comparison of arch length, AAO, DAo diameters and PWV $\mathrm{PRI}_{\mathrm{MI}}$ obtained with the ARTFUN approach and the proposed 3D method. p-values are computed for comparison between methods using a Kruskall-Wallis test, $r$ is the Pearson correlation coefficient and mean bias [limits of agreement] are given for the Bland-Altman analysis. 
Intra-observer reproducibility of the 3D measurements revealed mean absolute error (MAE) and coefficient of variations ( $\mathrm{CoV}$ ) of $5 \mathrm{~mm}$ and $3.7 \%$ respectively for the aortic arch length, $0.6 \mathrm{~mm}$ and $2.4 \%$ for the AAo diameter, $0.4 \mathrm{~mm}$ and $2.8 \%$ for the DAo diameter and $0.3 \mathrm{~m} / \mathrm{s}, 2.8 \mathrm{~mL}$ and $9.8 \%$ for the AAo volume, $2.7 \mathrm{~mL}$ and $6.4 \%$ for the DAo volume and $4.2 \%$ for the $P W V_{M R I}$.

\subsection{Effect of spatial resolution}

The mean Dice coefficient when considering the segmentation results at full resolution as reference were 0.910 (factor 2 ) and 0.886 (factor 3). Arch length variations were lower than 2 voxels (MAE: 4mm, CoV: $3 \%$ ) and AAo and DAo diameters approximately varied by 1 voxel (MAE $<2 \mathrm{~mm}$, CoV $<$ 6.2\%) for both subsampling factors. Although the variations were higher for AAo volume (scale 2: $\mathrm{MAE}=4.5 \mathrm{~mL}, \mathrm{CoV}=14 \%$; scale $3: \mathrm{MAE}=5.7 \mathrm{~mL}, \mathrm{CoV}=18 \%$ ) and for DAo volume (scale 2: MAE = 4. $2 \mathrm{~mL}, \mathrm{CoV}=8.3 \%$; scale $3: \mathrm{MAE}=6.1 \mathrm{~mL}, \mathrm{CoV}=11 \%$ ), such volume differences remained smaller than the volume increase in hypertensive patients as compared to normotensives (AAo: $+11 \mathrm{~mL}$, $+50 \%$; DAo: $+13 \mathrm{~mL},+33 \%)$.

\subsection{Associations of the measurements with age and cf-PWV}

The association between the 3D aortic measurements with age are shown in Fig.5 and summarized in Table 3. As expected, aortic length, diameters, volumes and $\mathrm{PWV}_{\mathrm{MRI}}$ from the $3 \mathrm{D}$ method increased linearly with age: $+9 \mathrm{~mm} /$ decade for aortic arch length; $+1.2 \mathrm{~mm} /$ decade and $+1.0 \mathrm{~mm} /$ decade for AAo

\begin{tabular}{ccccc}
\cline { 2 - 4 } & \multicolumn{2}{c}{ 3D } & \multicolumn{2}{c}{ ARTFUN } \\
\hline Arch length & $r=0.60$ & $p<0.001$ & $r=0.47$ & $p<0.001$ \\
AAo diameter & $r=0.48$ & $p<0.001$ & $r=0.46$ & $p<0.001$ \\
DAo diameter & $r=0.46$ & $p<0.001$ & $r=0.39$ & $p<0.001$ \\
PWV $_{\text {MRI }}$ & $r=0.64$ & $p<0.001$ & $r=0.63$ & $p<0.001$ \\
AAo volume & $r=0.50$ & $p<0.001$ & & \\
DAo volume & $r=0.49$ & $p<0.001$ & &
\end{tabular}

Table 3: Correlation between aortic measurements and age 
a)

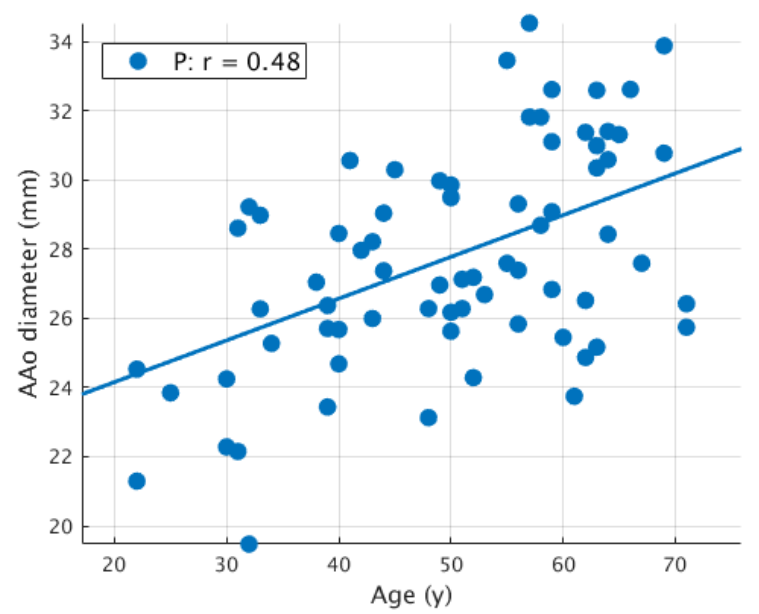

c)

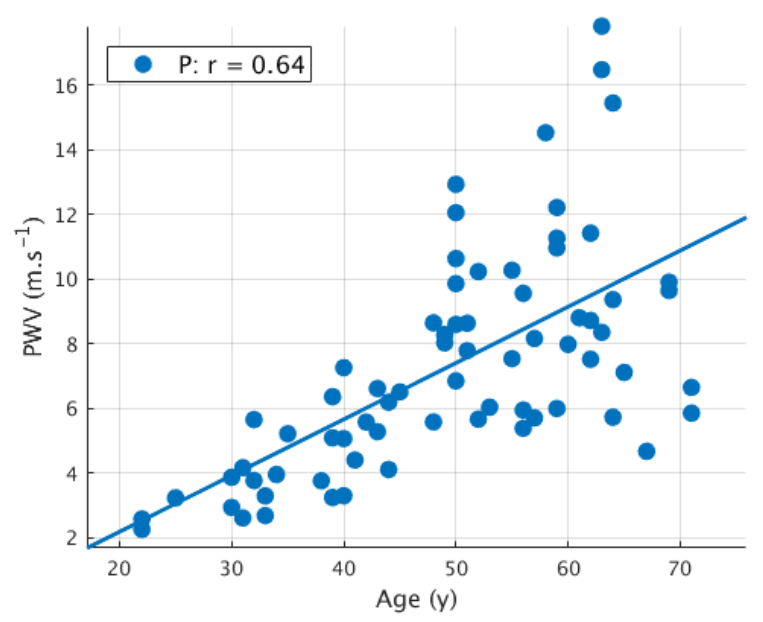

b)

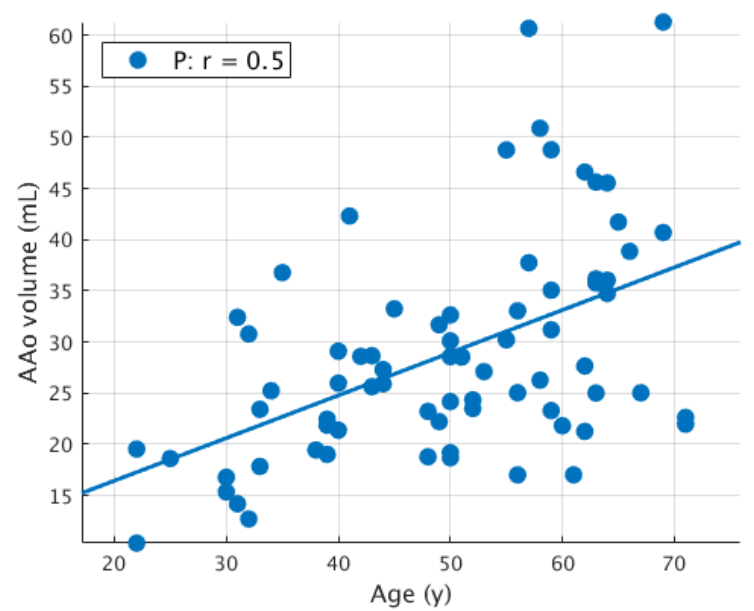

d)

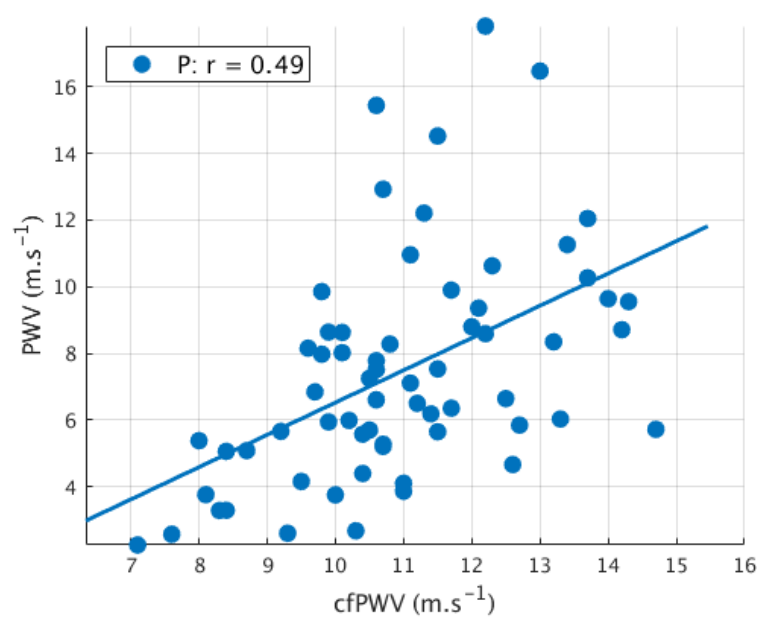

Figure 5: Associations of ascending (AAo) diameter (a) and volume (b) with age and of $\mathrm{PWV}_{\mathrm{MRI}}$ with (c) age and (d) cf-PWV.

and DAo diameters; $+2.6 \mathrm{~mL} /$ decade and $+4 \mathrm{~mL} /$ decade for AAo and DAo volumes and $+1.7 \mathrm{~m} . \mathrm{s}^{-}$ $1 /$ decade for $P W V_{M R l}$. Significant associations were found in all parameters $(p<0.001)$, with the highest correlation found between $P W V_{\text {MRI }}$ and age $(r=0.64)$. Such correlations remained significant after adjustment for gender, BMI and central SBP $(p<0.001)$. The association between PWV $\mathrm{VRI}_{\text {RI }}$ and cf-PWV (Fig. 5d) was also significant $(r=0.49, p<0.001)$. Stronger associations with age were found for volumes compared to diameters. Finally, similar or lower associations were found when considering the 2D ARTFUN indices. 

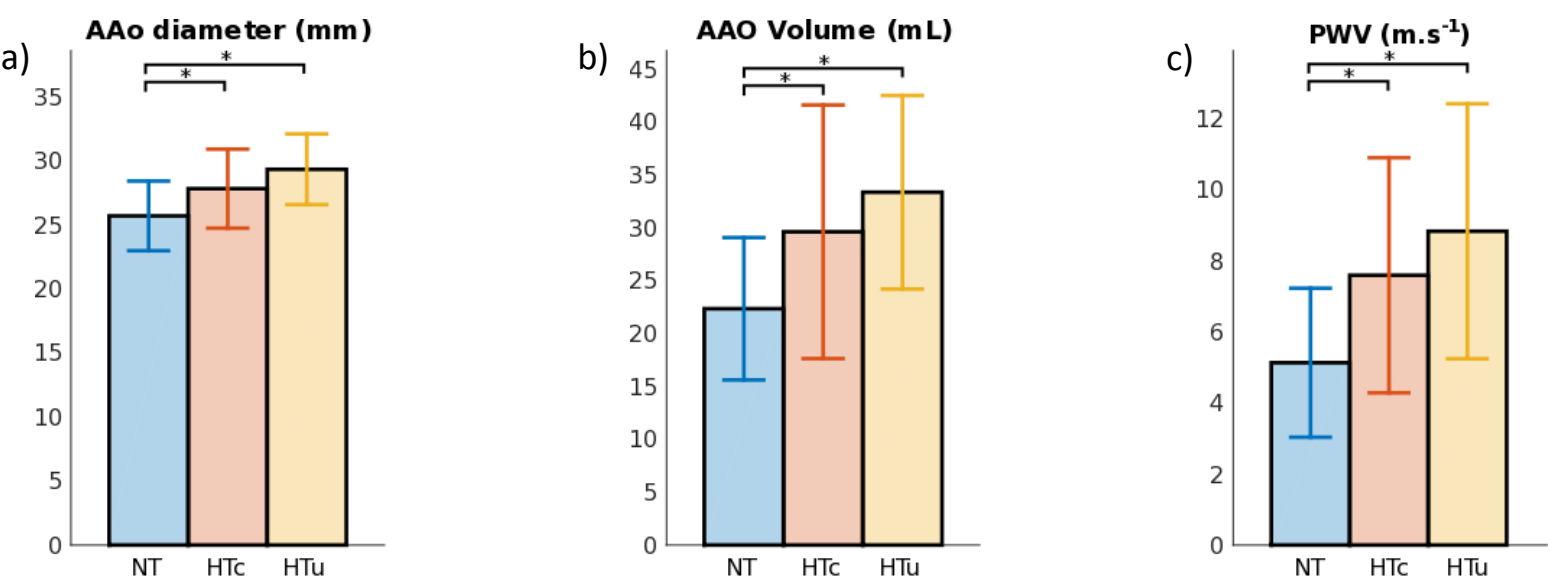

Figure 6: Differences between normotensives and hypertensives in term of (a) ascending aorta (AAO)

diameter, (b) $\mathrm{AAO}$ volume and (c) $\mathrm{PWV}_{\mathrm{MRI}}$ using the proposed method. Blue: normotensives (NT); red: controlled hypertensives (HTc) and yellow: uncontrolled hypertensives (HTnc). * means $p<0.05$

\subsection{Differences in aortic morphology related to hypertension}

Variations of the 3D aortic measurements with the presence of hypertension are illustrated in Figure

6. A significant increase across groups was found for all geometrical and stiffness measurements $(p<0.05$, Table 1). The pairwise comparisons between controlled or uncontrolled hypertensives with controls confirmed such significant increase except for aortic arch length and the DAo volume of controlled hypertensives which were higher than those of the control group, although the differences did not reach statistical significance. These results remained significant after diameter and volume indexation to BSA, except for the AAo diameter when considering comparison between controlled hypertensives and healthy controls.

\section{Discussion}

A fast semi-automated method for the reproducible segmentation of the aorta from 3D MRI data was described and used for the extraction of aortic morphological indices such as arch length, aortic diameters and volumes as well as PWV.

The consistency of our 3D measurements was demonstrated through: 1) the comparison of our measurements against well-established 2D indices which resulted in small mean biases and high correlations, 2) a good intra-observer reproducibility as well as low variations when decreasing the 
spatial resolution, 3) the high correlation with age and the reference cf-PWV, 4) the increase in aortic dimensions and stiffness in presence of hypertension.

An elongation of the aortic arch by $9 \mathrm{~mm} /$ decade and a dilation of the ascending (respectively descending) aorta by $1.2 \mathrm{~mm} /$ decade (respectively $1.0 \mathrm{~mm} /$ decade) were observed. Overall, the measured values found in this study were consistent with those reported in the literature $[1,7,26,27]$ although a direct comparison was not possible because of differences in measurement methods (2D on a single sagittal oblique view vs. 3D), patients age and cardiovascular risk.

Stronger associations with age were found for our measurements compared to ARTFUN, especially for the aortic arch length and DAo diameter. This latter finding might be explained by the fact that our 3D diameter measurement is less hampered by plane orientation or obliquity than a 2D measurement. While in 2D an acquisition plane is positioned to be as perpendicular as possible to both the AAo and DAo, in 3D diameters are computed in planes perpendicular to the centerline for every anatomical location.

Regarding the aortic arch length, our results were in line with those of a previous work [28] which compared $2 \mathrm{D}$ and $3 \mathrm{D}$ measurements and reported length differences $<10 \mathrm{~mm}$ that induced small variations in $\mathrm{PWV}_{\text {MRI }}\left(<0.5 \mathrm{~m} \cdot \mathrm{s}^{-1}\right)$, although smaller differences were found in our study (differences in length $<3 \mathrm{~mm}$ and $\mathrm{PWV}<0.25 \mathrm{~m} / \mathrm{s}$ ). This may be explained by the fact that in our $2 \mathrm{D}$ manual aortic centerline delineation strategy, a stack of axial views was used in addition to the $2 \mathrm{D}$ sagittal oblique view as reported in [28], to better account for aortic tortuosity [9]. However, even this multi-slice tracing strategy could not fully capture the local tortuosity as revealed by the BA plot (Figure 4.a), where the difference between the 2D and 3D methods seems to increase with the elongation of the aorta. As the aorta lengthens, it becomes more tortuous rendering its tracing by multi-slice 2D approach difficult and thus leading to higher differences between 2D and 3D derived lengths. These variations in length subsequently lead to higher differences in PWV as illustrated by the BA plot (Figure 4.d). 
A first limitation of our study is that since only local information was considered for the segmentation, a poor contrast could force the surface to remain close to the initialization as mean intensities inside and outside the interface are close. Although this can be solved by adding a small balloon force to move the surface outwards, it would require an additional tuning which would increase measurement variability.

Another limitation lies in the heterogeneity of the studied population which included patients with hypertension and healthy controls. This may explain the more modest strength of association with age and some of the differences in geometric values, as compared to other healthy populations studies reported in the literature. Moreover, the 3D segmentation was only applied on normotensive volunteers and hypertensive patients. It will therefore be interesting to test its ability to segment the aorta in patients with more severe aortic disease and more complex aortic geometries. However the primary goal of this study was to test the consistency of our 3D measurements against wellestablished 2D indices and their associations with age, presence of hypertension and reference cfPWV.

Finally, indication of prophylactic aortic surgery is usually guided by the measurement of maximal diameters at different locations. However, $50 \%$ of patients with aortic dissection have maximal aortic diameters below the recommended threshold for surgery $[29,30]$. As aortic volumes integrate both aortic dilatation and elongation, they may be more sensitive to changes in aortic geometry and less hampered by slice orientation and obliquity. However, this evaluation would require patients with more complex geometry and local deformations rather than hypertensive patients in which the aortic dilation is homogeneous and the circular cross-sectional shape is preserved.

\section{Conclusion}

In this study, a semi-automated tool for regional 3D segmentation of the aorta from SSFP MRI data was proposed. This method provided accurate geometrical (length, diameters, volumes) and 
functional aortic parameters $\left(P W V_{M R I}\right)$ in normotensive and hypertensive individuals with high reproducibility. Significant correlations were also found with a well-established 2D semi-automated technique, ageing and carotid-femoral PWV.

\section{Acknowledgements}

We would like to acknowledge the ECOS-SUD project number A15S04 (France-Argentina) exchange grant and the FRM project ING20150532487 for funding part of the technical aspects of this study and the FRHTA for funding the acquisitions and clinical data generation. We would like to thank Olivier Bernard for fruitful discussions on methodological aspects and implementation of the algorithm. 


\section{References}

1. Redheuil A, Yu W-C, Mousseaux E, Harouni AA, Kachenoura N, Wu CO, et al. Age-related changes in aortic arch geometry: relationship with proximal aortic function and left ventricular mass and remodeling. J Am Coll Cardiol. 2011;58:1262-1270.

2. Ohyama Y, Ambale-Venkatesh B, Noda C, Chugh AR, Teixido-Tura G, Kim J-Y, et al. Association of Aortic Stiffness With Left Ventricular Remodeling and Reduced Left Ventricular Function Measured by Magnetic Resonance Imaging. Circ Cardiovasc Imaging. 2016;9.

3. Vlachopoulos C, Aznaouridis K, Stefanadis C. Prediction of Cardiovascular Events and AllCause Mortality With Arterial Stiffness: A Systematic Review and Meta-Analysis. J Am Coll Cardiol. 2010;55:1318-27.

4. Redheuil A, Wu CO, Kachenoura N, Ohyama Y, Yan RT, Bertoni AG, et al. Proximal Aortic Distensibility Is an Independent Predictor of All-Cause Mortality and Incident CV Events: The MESA Study. J Am Coll Cardiol. 2014;64:2619-29.

5. Redheuil A. Cardiovascular aging: Insights from local and regional measures of aortic stiffness using magnetic resonance imaging. Artery Res. 2014;8:66-72.

6. Turkbey E., Jain A, Johnson C, Redheuil A, Arai A., Gomes A., et al. Determinants and normal values of ascending aortic diameter by age, gender, and race/ethnicity in the MultiEthnic Study of Atherosclerosis (MESA). J Magn Reson Imaging. 2014;39:360--368.

7. Davis $A E$, Lewandowski AJ, Holloway CJ, Ntusi NA, Banerjee R, Nethononda R, et al. Observational study of regional aortic size referenced to body size: production of a cardiovascular magnetic resonance nomogram. J Cardiovasc Magn Reson. 2014;16:1--9.

8. O'Rourke MF, Staessen JA, Vlachopoulos C, Duprez D, Plante GE. Clinical applications of arterial stiffness; definitions and reference values. Am J Hypertens. 2002;15:426-444.

9. Dogui A, Redheuil A, Lefort M, De Cesare A, Kachenoura N, Herment A, et al. Measurement of aortic arch pulse wave velocity in cardiovascular MR: comparison of transit time estimators and description of a new approach. J Magn Reson Imaging. 2011;33:13211329.

10. Bell V, Mitchell WA, Sigurosson S, Westenberg JJM, Gotal JD, Torjesen AA, et al. Longitudinal and Circumferential Strain of the Proximal Aorta. J Am Heart Assoc. 2014;3:e001536.

11. Wentland AL, Grist TM, Wieben O. Review of MRI-based measurements of pulse wave velocity: a biomarker of arterial stiffness. Cardiovasc Diagn Ther. 2014;4:193--206.

12. Bargiotas I, Mousseaux E, Yu W-C, Venkatesh BA, Bollache E, De Cesare A, et al. Estimation of aortic pulse wave transit time in cardiovascular magnetic resonance using complex wavelet cross-spectrum analysis. J Cardiovasc Magn Reson. 2015;17:1-11. 
13. Bollache E, Kachenoura N, Bargiotas I, Giron A, De Cesare A, Bensalah M, et al. How to estimate aortic characteristic impedance from magnetic resonance and applanation tonometry data? J Hypertens. 2015;33:575-583.

14. Barker AJ, Lanning C, Shandas R. Quantification of hemodynamic wall shear stress in patients with bicuspid aortic valve using phase-contrast MRI. Ann Biomed Eng. 2010;38:788800 .

15. Bensalah M, Bollache E, Kachenoura N, Giron A, De Cesare A, Macron L, et al. Geometry is a major determinant of flow reversal in proximal aorta. Am J Physiol-Heart Circ Physiol. 2014;1408-16.

16. Potters WV, van Ooij P, Marquering H, vanBavel E, Nederveen AJ. Volumetric arterial wall shear stress calculation based on cine phase contrast MRI. J Magn Reson Imaging. 2015;41:505-516.

17. Sigovan M, Dyverfeldt P, Wrenn J, Tseng EE, Saloner D, Hope MD. Extended 3D approach for quantification of abnormal ascending aortic flow. Magn Reson Imaging. 2015;33:695700 .

18. Barbosa DC, Dietenbeck T, Schaerer J, D’hooge J, Friboulet D, Bernard O. B-Spline Explicit Active Surfaces: An efficient framework for real-time 3D region-based segmentation. IEEE Trans Image Process. 2012;21:241-251.

19. Uitert RV, Bitter I. Subvoxel precise skeletons of volumetric data based on fast marching methods. Med Phys. 2007;34:627-38.

20. Hassouna MS, Farag AA. MultiStencils Fast Marching Methods: A Highly Accurate Solution to the Eikonal Equation on Cartesian Domains. IEEE Trans Pattern Anal Mach Intell. 2007;29:1563-74.

21. Lankton S, Tannenbaum A. Localizing Region-Based Active Contours. IEEE Trans Image Process. 2008;17:2029-2039.

22. Barbosa D, Heyde B, Cikes M, Dietenbeck T, Claus P, Friboulet D, et al. Real-time 3D interactive segmentation of echocardiographic data through user-based deformation of Bspline explicit active surfaces. Comput Med Imaging Graph. 2014;38:57-67.

23. Dogui A, Kachenoura N, Frouin F, Lefort M, De Cesare A, Mousseaux E, et al. Consistency of aortic distensibility and pulse wave velocity estimates with respect to the Bramwell-Hill theoretical model: a cardiovascular magnetic resonance study. J Cardiovasc Magn Reson. 2011;13:1-8.

24. Herment A, Kachenoura N, Lefort $M$, Bensalah $M$, Dogui A, Frouin F, et al. Automated Segmentation of the Aorta from Phase Contrast MR Images: Validation Against Expert Tracing in Healthy Volunteers and in Patients with a Dilated Aorta. J Magn Reson Imaging. 2010;31:881--888. 
25. Herment A, Lefort M, Kachenoura N, Cesare AD, Taviani V, Graves MJ, et al. Automated Estimation of Aortic Strain From Steady-State Free-Precession and Phase Contrast MR Images. Magn Reson Med. 2011;65:986-993.

26. Hickson SS, Butlin M, Graves M, Taviani V, Avolio AP, McEniery CM, et al. The relationship of age with regional aortic stiffness and diameter. JACC Cardiovasc Imaging. 2010;3:1247-1255.

27. Kawel-Boehm N, Maceira A, Valsangiacomo-Buechel ER, Vogel-Claussen J, Turkbey EB, Williams $R$, et al. Normal values for cardiovascular magnetic resonance in adults and children. J Cardiovasc Magn Reson. 2015;17:29.

28. van Engelen A, Silva Vieira M, Rafiq I, Cecelja M, Schneider T, de Bliek H, et al. Aortic length measurements for pulse wave velocity calculation: manual $2 \mathrm{D}$ vs automated $3 \mathrm{D}$ centreline extraction. J Cardiovasc Magn Reson. 2017;19:32.

29. Elefteriades JA, Farkas EA. Thoracic aortic aneurysm: clinically pertinent controversies and uncertainties. J Am Coll Cardiol. 2010;55:841-857.

30. Kallenbach K, Sundt TM, Marwick TH. Aortic Surgery for Ascending Aortic Aneurysms Under $5.0 \mathrm{~cm}$ in Diameter in the Presence of Bicuspid Aortic Valve. JACC Cardiovasc Imaging. 2013;6:1321-1326. 